\title{
Transitional cell carcinoma: a case for collaboration
}

\author{
R J Marshall, E F D Mackenzie, J Hill, L M Fearn
}

\begin{abstract}
Urine samples form the greatest proportion of the numerical workload of most clinical microbiology laboratories. During the screening by microscopy, atypical cells were detected which did not conform to the normal cellular morphology found in urinary deposits. These specimens were referred to the Department of Cytopathology for a second opinion. From these referrals a small but significant number of previously undiagnosed transitional cell carcinomas were detected.
\end{abstract}

Microbiology staff involved in the microscopic examination of urine samples have noticed for some time cells which were unusual or atypical. Such samples were referred to the Department of Cytopathology for identification of cell type. As it became apparent that this was a way of contributing to patient care by identifying possible malignancies, the procedure was formalised and now operates as described below.

\section{Methods}

Routine urine microscopy was performed using an inverted microscope with $60 \mu \mathrm{l}$ of urine in a microtitre well. Phase contract microscopy was not used. Any urine sample thought to contain atypical cells as defined by the Department of Cytopathology-that is, bizarre shapes; high nuclear cytoplasmic ratio; an atypical arrangement one to another-was referred to cytopathology. The sample was preserved by the addition of $1-2 \%$ of methanol if there was the possibility of a delay in the sample being prepared for cytological examination. Urine samples containing atypical cells in conjunction with overt urinary tract infection were excluded from referral.

Microbiology staff are encouraged to seek a second opinion within their department on any groups of cells thought to be atypical. Only cells thought to be suspicious by an experienced member of staff were referred. Members of the cytopathology staff were asked to give occasional lectures to microbiology staff on the recognition of relevant cells, and black and white photographic prints of atypical cells were made available alongside the "urine" microscope.

\section{Results}

During the period 1 January to 31 October 1990, 38 urine samples-17 from general practice, 16 from urology wards, and five from general medicine-were referred to the Department of Cytopathology. Eleven (29\%) gave an inadequate sample of cells, $16(42 \%)$ were negative for malignant cells, but $11(29 \%)$ showed malignant cells, seven from male patients and four from female patients. Positive samples came from general practice $(n=5)$, urology wards $(n=5)$, and from non-urology inpatients $(n=1)$. Four of the samples from urology wards were subsequently found to have come from patients with a previous clinical history of malignancy. The remaining seven samples that were positive for malignant cells were mainly from general practice patients with no previous history of malignancy. The age range of the patients with positive results was 64 to 90 years. It was estimated that 28-32 Welcan units were expended per specimen, or 20 hours over a period of one year.

\section{Comment}

It must be made clear that microbiology staff never comment to clinicians directly on the possibility of malignancy, and that all requests for repeat specimens and the clinical follow up are dealt with by the Department of Cytopathology. Referrals giving negative results are not reported.

Transitional cell carcinoma is reported to have a national prevalence of 384 cases/million population in the United Kingdom (National Cancer Research Institute, 1990). Given the large number of urine samples screened through a typical microbiology department it is inevitable that urine samples containing malignant cells are sometimes processed. The fact that in our experience a number of these malignancies were previously undiagnosed offers the opportunity for increasing overall patient care for a small effort in the laboratory. 